\title{
Generating high-avidity human Mabs in mice
}

\author{
Michael Neuberger
}

On page 845 of this issue, Fishwild et al. describe the isolation of high-avidity monoclonal antibodies (Mabs) specific for human CD4 that have been obtained from mice transgenic for human immunoglobulin gene miniloci ${ }^{1}$. The novelty of the report is not that minilocus-transgenic mice have been used for the isolation of human antibodies to human antigens, but rather that the antibodies elicited are of high avidity $\left(\mathrm{K}_{\mathrm{a}} 10^{9}-10^{10}\right.$ $\mathrm{M}^{-1}$ ) and that they derive from more than one B-cell lineage. These results constitute a significant step forward in demonstrating the usefulness of minilocus-transgenic mice for the isolation of human Mabs.

There are now several approaches to the isolation of human Mabs-minilocus transgenics, phage display, and in vitro culture of human lymphocytes. In all cases, the isolation of specific Mabs essentially comes down to a two-stage process: First, the generation of a repertoire of Mabs with distinct specificities; and second, selection of those Mabs out of the repertoire that bind the antigen. It is important that the antibodies isolated have good binding characteristics because the main purpose of making human Mabs is to use them in humans, either to target tissues or to bind and neutralize foreign substances. The latter, of course, represents the physiological function of the humoral immune system and, to this end, animals have elaborated sophisticated processes to ensure that the antibodies elicited are of high affinity.

In the transgenic approach, mouse lines are created that, in essence, contain human immunoglobulin gene miniloci instead of the corresponding mouse loci. The intention is to exploit the normal diversification/selection process of the mouse immune system in order to obtain highaffinity human antibodies.

This diversification/selection process can be divided into two phases. First, diversification of the primary repertoire is achieved by V-D-J joining (Fig. 1). The essential feature of the resulting primary repertoire is not so much that it yields antibodies with high binding constants, but rather that it contains a wide enough range of specificities to ensure that any invading antigen will find at least one antibody that recognizes it sufficiently well to initiate an immune response.

Michael Neuberger is a member of the scientific staff of the MRC Laboratory of Molecular Biology, Hills Road, Cambridge CB2 2QH, UK (msn@mrc-lmb.cam.ac.uk).
Second, once this is achieved, another wave of diversification takes place in which frantic proliferation is accompanied by hypermutation of the antibody genes. This produces a secondary repertoire in which antibodies selected from the primary repertoire are further diversified by the nearly random introduction of amino acid substitutions throughout the V segment. Antigen
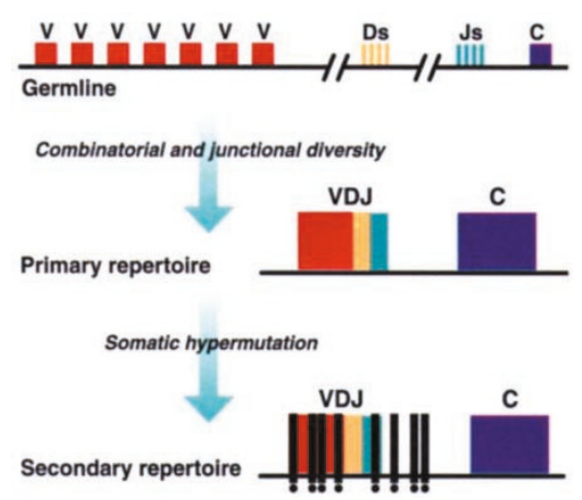

Figure 1. High-affinity antibodies are made by a two-stage process: gene rearrangement and somatic hyprtmutation.

selection then ensures that those secondary antibodies with good kinetic parameters dominate the response.

Early studies have revealed that human germline-configuration immunoglobulin gene segments introduced into mouse germline will rearrange and produce a functional primary repertoire ${ }^{2}$. Although human immunoglobulin from this primary repertoire can be selected following antigen challenge, the process is made much more effective using strains that carry disruptions of the endogenous mouse immunoglobulin gene loci (essentially removing the competi- tion between the introduced human and endogenous mouse antibody repertories $)^{3-5}$. Using this approach, the only way for the mouse to respond to antigen challenge is to produce human antibodies. The fact that minilocus transgenics can respond to a variety of antigens ${ }^{3-5}$, together with the significant finding that, on challenge with CD4, they can elicit more than one lineage of antibodies (as judged by $\mathrm{V}$ gene usage) ${ }^{1}$, indicates that the primary repertoire generated in these animals is indeed sufficiently diverse to be useful for isolating antibodies against a wide variety of antigens.

The next issue is whether the antibodies elicited will be of high affinity. This will depend on whether the secondary wave of diversification/selection occurs satisfactorily. Other studies by the GenPharm group ${ }^{6}$, as well as by ourselves, indicate that the $\mathrm{V}$ genes of some of the miniloci created indeed constitute effective targets for the somatic hypermutation process (although it is difficult to quantify whether this mutation is occurring with full vigor). Therefore, it may be anticipated that the CD4-specific antibodies isolated by Fishwild et al. will have had their affinity fashioned by somatic mutation; it would be good to see this confirmed. Nevertheless, the proof of the pudding is in the eating; The results of Fishwild et al. provide powerful support for the usefulness of the transgenic minilocus technology.

1. Fishwild, D.M. et al. 1996. Nat. Biotechnol. 14:845851.

2. Bruggemann, M. et al. 1989. Proc. Natl. Acad. Sci. USA 86:6709-6713.

3. Lonberg, N. et al. 1994. Nature 368:856-859.

4. Green, LL. et al. 1994. Nat. Genet. 7:13-21.

5. Wagner, S.D. et al. 1994. Eur. J. Immunol. 24:26722681.

6. Taylor, L.D. et al. 1993. Int. Immunol. 6:579-591.

7. Wagner, S.D. et al. 1996. Genomics. In press.

\section{Suicidal tumor proteases}

\section{Bonnie F. Sloane}

Numerous studies have linked proteolytic enzymes to malignant progression, invasion, and metastasis. This is true for the following: matrix metalloproteases (MMPs), such as

Bonnie F. Sloane is professor and chair of the department of pharmacology at Wayne State University, Detroit, MI 48201-1908

(bsloane@cms.cc.wayne.edu). gelatinases $\mathrm{A}$ and $\mathrm{B}$; stromelysin and matrilysin; serine proteases, such as urokinase; aspartic proteases, such as cathepsin D; and cysteine proteases, such as cathepsins B and L. Readers interested in recent review articles on this topic should consult the issue of Enzyme \& Protein on "proteolytic enzymes in cancer invasion"'.

Tumor cells were long thought to be the primary source of proteases within a tumor. 